\title{
Prediction of Mechanical Properties of Ti-6Al-4V using Neural Network
}

\author{
Detak Yan Pratama ${ }^{1, a}$, Syarif Junaidi ${ }^{2, b}$ and Ramli R. ${ }^{3, c}$ \\ ${ }^{1,2,3}$ Dept. of Mechanical and Materials Eng. Universiti Kebangsaan Malaysia, Malaysia \\ adetak@vlsi.eng.ukm.my, 'syarif@vlsi.eng.ukm.my, 'rizauddin@vlsi.eng.ukm.my
}

Keywords: Ti-6Al-4V, Mechanical Properties, Neural Network, Prediction, Learning Algortihm.

\begin{abstract}
Objective of this study is to develop simulation for predicting mechanical properties of Ti-6Al-4V alloy. Rockwell Hardness (HRC), Ultimate tensile strength (UTS) and elongation ( $\varepsilon$ ) are predicted by using Neural Network (NN) with multilayer feedforward architecture. The input of simulations are chemical compositions of Ti-alloy at room temperature. The data of the mechanical properties which are reported by other researchers are used for the NN training and Gradient Descent (GD) and Lavenberg Marquardt (LM) are applied as methods of learning algorithms. The results of training by both methods are compared in order to obtain high performance of output criteria which are determined by a Normalized Root Mean Square Error (NRMSE). is used to determine the performance of output criteria. In training, the NRMSE output calculated by GD algorithm show that HRC, UTS and $\varepsilon$ are $0.024,0.0717$ and 0.1375 respectively, while LM algorithm for HRC, UTS and $\varepsilon$ are $0.0207,0.0689$ and 0.1150 , respectively. The NRMSE predicted output of GD algorithm for HRC, UTS, and $\varepsilon$ are $0.0658,0.0338$ and 0.2994 , while LM algorithm for HRC, UTS and $\varepsilon$ are $0.0371,0.1192$ and 0.5487 respectively. In training, values of NRMSE calculated by LM algorithm is smaller than GD algorithm. These results suggest that LM algorithm shows excellent ability for training, however the GD method is more appropriate for the training algorithm in order to obtain a high performance of output criteria. It can be concluded that the NN can be applied for predicting mechanical properties of Ti-6Al-4V alloys.
\end{abstract}

\section{Introduction}

Ti-6Al-4V is one type of titanium alloys which is used in many application such as biomedical and aerospace [1]. The properties of Ti-6Al-4V depends on material compositions and heat treatment. Since titanium is an expensive material, experimental study, which is needed to obtain the mechanical properties, will cause high cost. Thus, a simulation for predicting suitable mechanical properties is needed to reduce cost for experiment.

Prediction of mechanical properties by using Neural Network (NN) has been developed by other researcher $[2,3,4]$, but none of them described about mechanical properties of Ti-6Al-4V. A NN is thought to be one of the simulation technique which is an effective for the prediction of mechanical properties. The principle of the technique resembles the human brain in two aspects: the knowledge is acquired by the $\mathrm{NN}$ through a learning process and inter-neuron connection strengths, known as synaptic weights, are used to store the knowledge.

Most of the NN simulation is built only for predicting one output object [2]. Also, simulation for many output have been achieved, the training process is carried out separated [3]. In this study, prediction system using NN for predicting mechanical properties of Ti-6Al-4V as a whole of Rockwell Hardness (HRC), Ultimate Tensile Strength (UTS) and elongation ( $\varepsilon$ ) based on material composition is developed. 


\section{Model Description}

\section{Fundamental Neural Network}

$\mathrm{NN}$ models can be used as an alternative method for analysis and predictions in engineering. NN mimics learning process of human brain. The operation is like a "black box" model, and requires no detailed information about the system. Moreover, it can learn the relation between the input parameters and controlled and uncontrolled variables by studying previously recorded data, in a way similar to how a non-linear regression might be performed. Other advantage of $\mathrm{NN}$ is the ability to handle large and complex systems with many interrelated parameters. It can ignore excessive data that have low significance, and concentrate only on important inputs [5].

NN usually consists of an input layer, hidden layers and an ouput layer. Each single neuron is connected to other neurons from previous layer through adaptable synaptic weights. Knowledge is usually stored as a set of connection weights that corresponds to synapse-efficacy in biological neural systems.

In NN, training is a process of modifying the connection weights in some orderly fashion by using a suitable learning method. Actually, there are two types of $\mathrm{NN}$ training, supervised and unsupervised [6]. This study discuss $\mathrm{NN}$ with supervised training especially backpropagation algorithm. The NN uses a learning mode, in which an input is presented to the NN attempts to produce the desired output. The weight after training contains meaningful information whereas before training they are random and have no meaning.

Fig. 1 illustrates the NN that is used for the prediction system. There are 8 nodes in input layer, 20 nodes in hidden layer and 3 nodes in output layer. Each node from the input layer is connected to all nodes in hidden layer, and the nodes in hidden are connected to output layer. Each weighted input signal for applying an output is summed by Each node in hidden layer. Before applying an output, the summing result passed through an activation function. All neurons in the same layer usually have same activation function, although it is not required. This prediction system uses binary sigmoid refers with Eq.1,

$$
f(x)=\frac{\exp (x)-\exp (-x)}{\exp (x)+\exp (-x)},
$$

for activation function in hidden layer and linier function refers to Eq. 2 for the output nodes.

$$
f(x)=x
$$

For outgoing connection, the activation value is multiplied by the specific weight and transferred to next node.

There are three principle parts that must be identified to produce the reliable NN. They are architecture, learning algorithm and transfer function. NN will run well if best combination between is found, but there is no exact rule for this. Thus, number of node in hidden layer should be adjusted for 20 nodes.

\section{Input and Output Data Pairs}

Pairs data input and output are needed in order to obtain reliable NN. In this paper, the input of NN are material compositions of Ti-6Al-4V. There are 8 eight main chemical compositions are applied used to train the NN. The output data are HRC, UTS and $\varepsilon$. All data pairs are obtained from datasheet of material properties. 


\section{Learning Algorithm}

It is known that one of the important principle to build $\mathrm{NN}$ is a learning algorithm. This prediction system will compare two type of learning algorithm, i.e., Gradient Descent (GD) and Lavenberg Marquardt (LM). Both of the learning algorithm are backpropagation algorithm. Training NN by

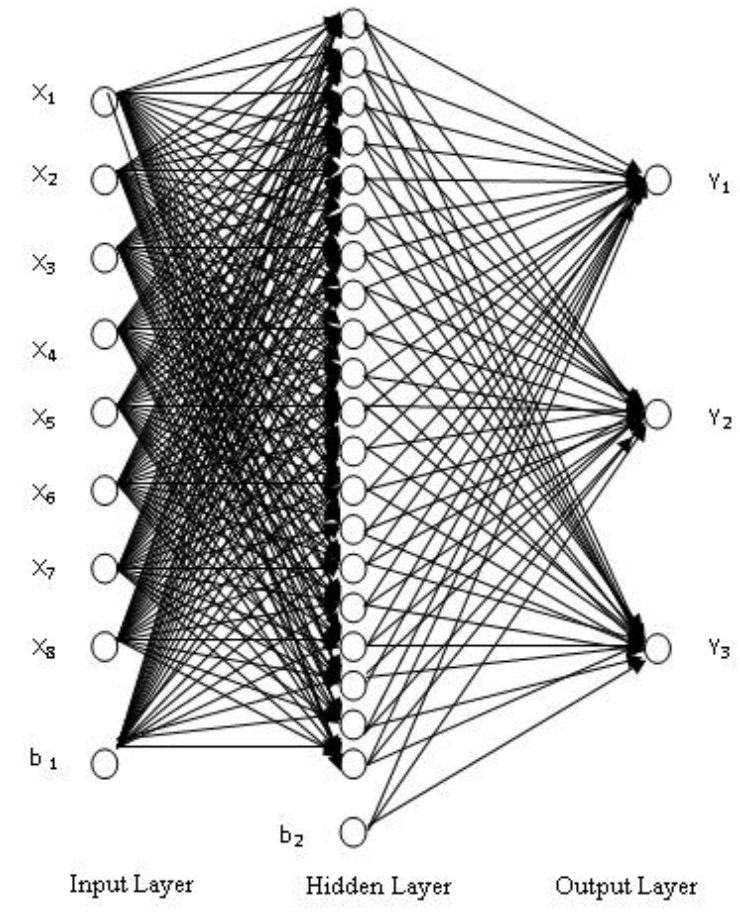

Fig. 1. Information processing in a NN unit.

backpropagation involves three stages that is feedforward of the input training pattern, backpropagation of the associated error and adjustment of the weights. GD represents algorithm that uses gradient to reduce the error while LM represents the hessian to reduce the error.

Generally NN works by training input and output data pairs to adjust the weights. The adjusted weights are applied to test the NN to make a prediction. The $\mathrm{NN}$ will reliably run based on accuracy of the weight. Accurate weights can be adjusted by the NN training as parameter set. Through this training part, the $\mathrm{NN}$ recognized the data and create new weight.

In order to make sure that the $\mathrm{NN}$ has excellent performance, it is known that comparing the data output target and output from the $\mathrm{NN}$ can improve performance the NN. The difference between these outputs can be calculated and is known as Normalized Root Mean Square Error (NRMSE) as follows on Eq.3,

$$
N R M S E=\sqrt{\frac{\sum[y(t)-y(p)]^{2}}{\sum[y(t)]^{2}}},
$$

where, $y(t)$ is output target that adjust as pairs of output data, a $y(p)$ is the output produced by the NN.

\section{Result and Discussions}

\section{Neural Network Training}

This NN is trained by using two learning algorithm, GD and LM. Both of them are applied to predict HRC, UTS and $\varepsilon$ based on the material composition of alloy.

Fig. 2 shows NRMSE for training part of a NN using GD and LM. Generally, NRMSE of GD are higher than LM algorithm in all output nodes. This fenomena is caused the algorithm of LM is more 
complicated than GD, so the error degradation of LM is faster than GD. These graphics represent NRMSE data for all adjusting node in hidden layer in order to obtain the proper number of node for achieving excellent performance. The lowest accumulation of NRMSE data is on amount of node 19. For these nodes, the NRMSE training of HRC is 0.024 , UTS is 0.0717 and $\varepsilon$ is 0.1375 by using GD algorithm. While LM algorithm shows 0.0207 for HRC, 0.0689 for UTS and 0.1150 for $\varepsilon$.

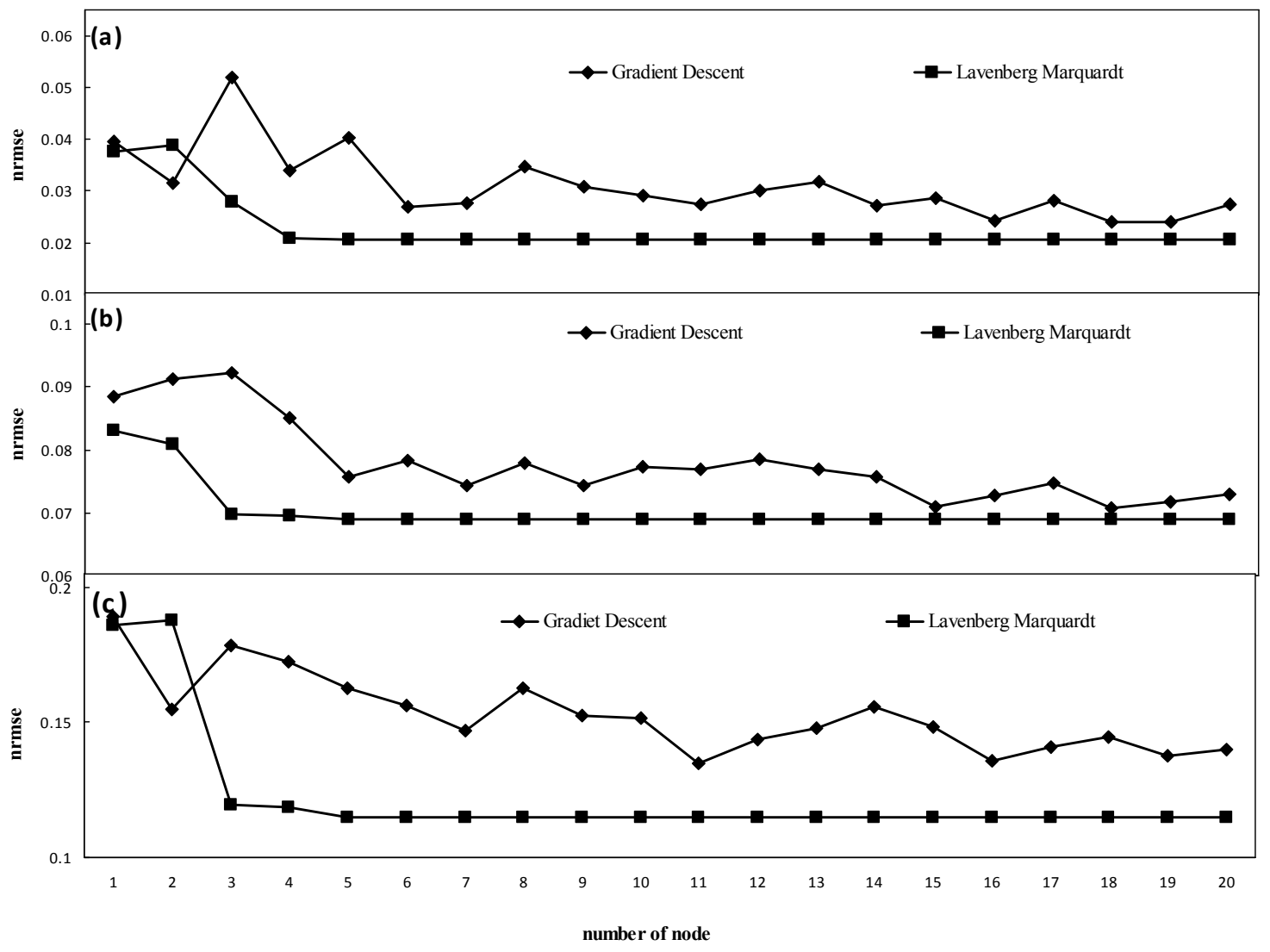

Fig. 2. NRMSE training for HRC (a), UTS (b) and $\varepsilon$ (c) output.
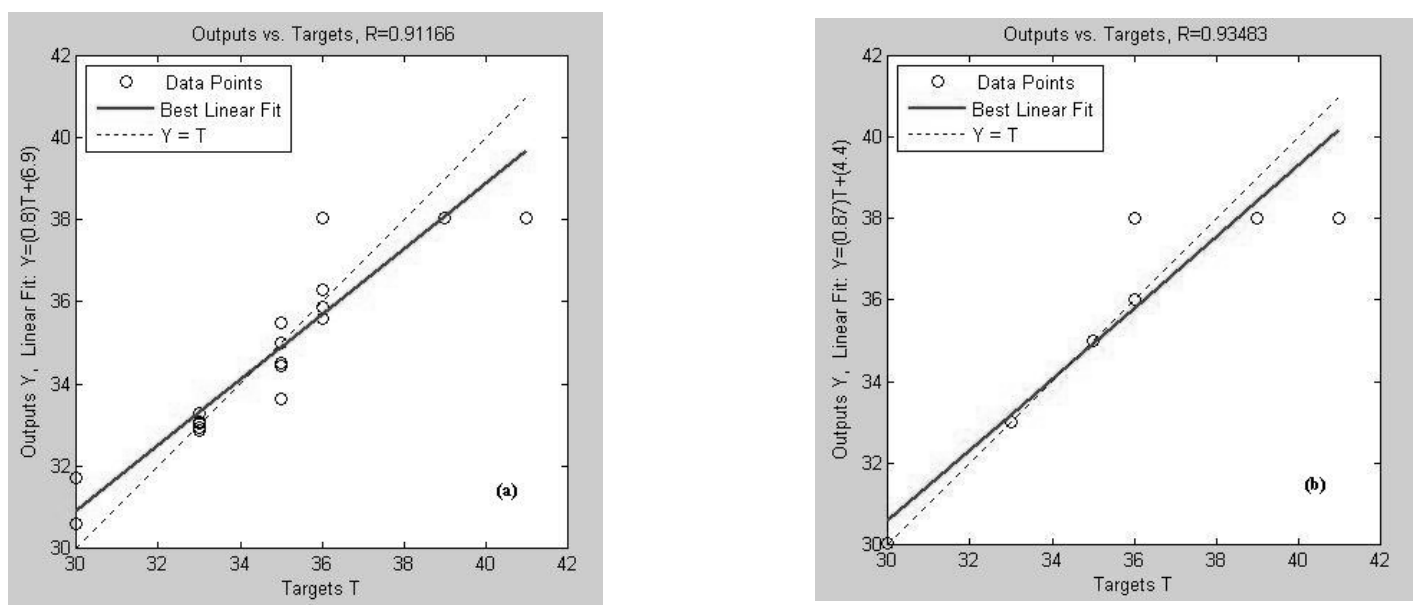

Fig. 3. Performance of the NN in training part using GD algorithm (a) and LM algorithm (b).

Performance of NN in training using GD and LM algorithms can be shown in Fig.3. Training data pairs are trained for whole output nodes. Coefficient correlation (R) values show linear regression between output target and output produced by the $\mathrm{NN}$. The values show degree of accuracy of the NN. The R of GD algorithm is 0.9116 which is smaller than that of LM algorithm; 0.93483. It means that the degree of accuracy in the LM algorithm in training NN is better than GD algorithm. Thus, LM algorithm which has more complex algorithm than GD algorithm, gives more compatible result for training input and output data pairs of $\mathrm{NN}$. 


\section{Neural Network Simulation}

Different data pairs of input and output are simulated on two types of learning algorithm, GD and LM. Fig. 4 shows NRMSEs simulation part of NN. It is found that the NRMSE from training differ from the value obtained by simulation. NRMSE obtained by the GD algorithm are smaller than that obtained by LM algorithm. Moreover, the smallest NRMSE value of output is on amount of 19 nodes. The NRMSE simulation using GD algorithm of HRC, UTS and $\varepsilon$ are $0.0658,0.0338$, and

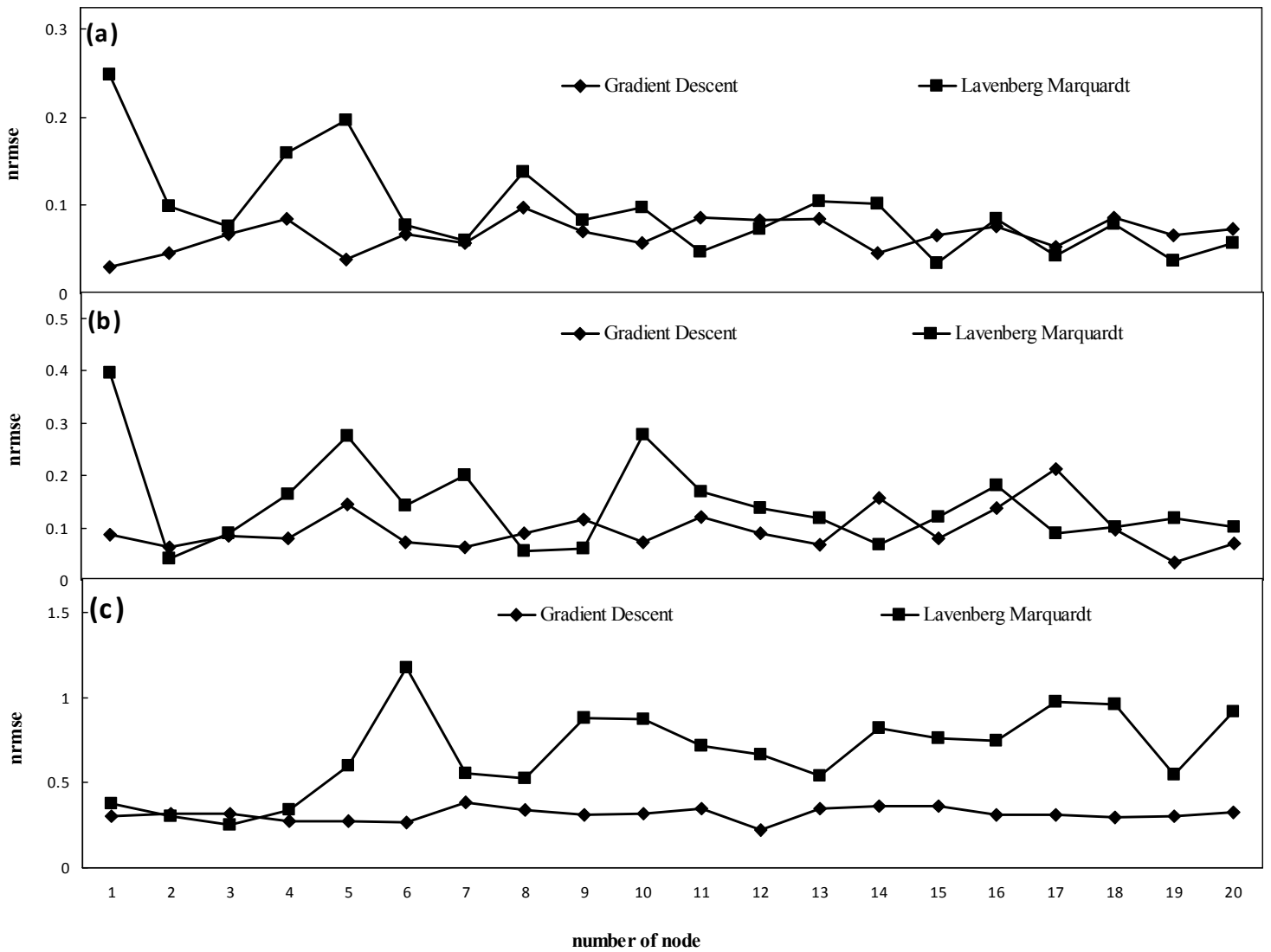

Fig. 4. NRMSE simulation for HRC (a), UTS (b) and $\varepsilon$ (c) output.
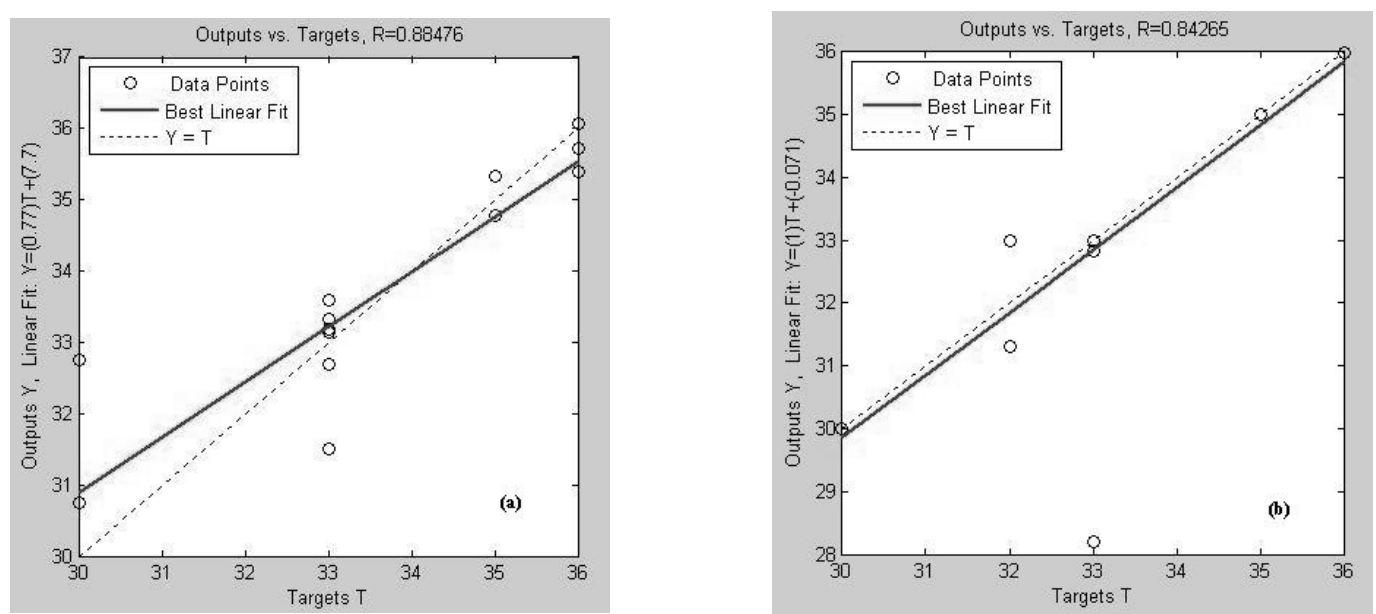

Fig. 5. Performance of the NN in simulation part using GD algorithm (a) and LM algorithm (b). 
0.2994, respectivel. On the other hand, NRMSE from the LM algorithm of HRC, UTS and $\varepsilon$ are $0.0371,0.1192$ and 0.5487 , respectively.

Fig. 5 shows the R of GD is higher than that from LM algorithm. It means GD algorithm has better ability than LM algorithm in simulation. From these results, it can be concluded that more than one of mechanical properties of Ti alloy can be predicted by one simulation using the NN. It is found that the LM algorithm is suited for training. On the other hand, the GD algorithm is appropriate for simulation.

\section{Summary}

Whole of data simulation show that NRMSE of Gradient Descent algorithm is smaller than Lavenberg Marquardt algorithm. Gradient Descent algorithm is more compatible used to predict the mechanical properties of Ti-6Al-4V.

\section{References}

[1] G.Lutjering, J.C. Williams, in : Titanium, Engineering Materials and Process, Springer, Verlag Berlin Heidelberg 2003 (2007)

[2] Z. Guo, S. Malinov, W. Sha: Comput. Mat. Sci. 32 (2005), p. 1-12

[3] J. McBride, S. Malinov, W. Sha: Mat. Sci. Eng. A 384 (2004), p. 129-137

[4] Miaoquan Li, Xuemie Liu, Aiming Xiong: submitted to Journal of Material Processing Technology 121 (2002)

[5] Soteris A. Kalogirou: Appl Energy 67 (2000) p. 17-35

[6] Laurene Fausset, in: Fundamentals of Neural Networks, Prentice Hall, Englewood Cliffs (1994) 